\title{
Multiplexed, linkage group-specific SNP marker sets for rapid genetic mapping and fingerprinting of sugar beet (Beta vulgaris L.)
}

\author{
Silke Möhring ${ }^{1}$, Francesco Salamini ${ }^{1}$ and Katharina Schneider ${ }^{1,2, *}$ \\ ${ }^{1}$ Max Planck Institut für Züchtungsforschung, Carl-von-Linné Weg 10, 50829 Köln, Germany; ${ }^{2}$ Current \\ address: GSF Forschungszentrum, Ingolstädter Landstr. 1, 85764 Neuherberg, Germany; *Author for \\ correspondence (e-mail: katharina.schneider@gsf.de; phone: 0049-(0)89-3187-2203; fax: \\ 0049-(0)89-3187-3323)
}

Received 21 March 2004; accepted in revised form 21 June 2004

Key words: Beta vulgaris, Fingerprinting, Genetic mapping, Multiplex analysis, SNP, SNaPshot

\begin{abstract}
A set of single nucleotide polymorphism (SNP) markers has been developed for each of the nine linkage groups of sugar beet. Each set can monitor the polymorphic state at five to six linked marker loci. In each set, the loci selected for marker development are first amplified in a multiplexed reaction. These amplification products are the basis for sequence-specific elongation of primers adjacent to SNP positions. The extension step revealing SNP loci is based on fluorescently labelled nucleotides. In each set, primers developed to reveal SNP alleles differ in length to allow clear peak resolution in capillary electrophoresis. The nine linkage group (LG) - specific sets provide information on the polymorphism at a total of 52 SNP marker loci. Using the SNP-based tool, groups of concerned loci have been anchored to three different linkage maps of sugar beet. In a second experiment, sugar beet breeding lines have been fingerprinted. The use of the nine sets of LG-specific markers in sugar beet genetics and breeding is discussed. The information necessary to specify the 52 marker loci, as well as their map location, and all details concerning SNP assays, including allele type and nature of mutation, are reported.
\end{abstract}

Abbreviations: LG - linkage group; SNP - single nucleotide polymorphism; RFLP - restriction fragment length polymorphism; RAPD - random amplification of polymorphic DNA; AFLP - amplified fragment length polymorphism; CAPS - cleaved amplified polymorphic sequence; SSCP - single strand conformation polymorphism; SSR - simple sequence repeat; STS - sequence tagged site; QTL - quantitative trait locus; nt - nucleotide

\section{Introduction}

Molecular tools supporting sugar beet breeding have been developed particularly in Europe, where this crop is the major source for sucrose production. Currently, they mainly consist of dense molecular linkage maps based on RFLP, RAPD and AFLP markers, to which several expressed genes and few mutants have been associated (Barzen et al. 1992; Barzen et al. 1995; Schumacher et al. 1997; Schneider et al. 1999; Schneider et al. 2002; Hunger et al. 2003). Additionally, large insert libraries of YAC and BAC clones are available, and positional cloning was implemented to identify a gene conferring resistance to nematodes (Cai et al. 1997; Hohmann et al. 2003). QTLs for disease resistance traits, as well as sucrose quality and yield, were located on the genetic map, and candidate genes to be used in marker-assisted selection have been proposed (Schneider et al. 2002; Hunger et al. 2003). So far, genotyping in sugar beet was performed by RFLP filter hybridization and PCR-based RAPD, AFLP, CAPS and SSCP analyses using agarose and native polyacrylamide gels. However, increasing demand for determining single Men- 
delian factors or QTLs affecting monogenic or polygenic traits of agronomic importance necessitates the development of high throughput marker systems offering high accuracy for genotyping. Additionally, there is a need for DNA fingerprinting in plant breeding to identify unknown genotypes, protect intellectual property and test for seed purity. This is why the possibility of using a very abundant class of molecular markers, single nucleotide polymorphisms (SNPs), in sugar beet was raised in a recent paper (Schneider et al. 2001). In the sugar beet genome, SNPs were found at a rate of one every 130 base pairs, which corresponds to at least $5.4 \times 10^{6}$ heterozygous SNP loci when random crosses among currently used genotypes are considered. SNPs are genetically stable and codominant, and their analysis is amenable to partial or full automatization, thereby reducing significantly genotyping cost. Their potential in crop genetics and plant breeding has been reviewed by Rafalski (2002). To visualize the SNP alleles several technological platforms relying on chip hybridization, enzymatic cleavage, ligation assays or primer extension assays using fluorescence detection or mass spectrometry are available (reviewed in Syvanen 2001). Based on such premises, a project was started to provide sets of SNP markers specific for each of the nine linkage groups (LGs) of sugar beet. Genetically defined loci were selected for SNP marker development based on comparative DNA sequencing in a group of sugar beet breeding lines. Groups of five to six markers per LG were assembled, multiplexing both the initial marker locus amplification and the SNP scoring step. Scoring of the SNP alleles was performed by the primer extension-based SNaPshot ${ }^{\mathrm{TM}}$-minisequencing procedure (Applied Biosystems, Foster City, CA, USA). The paper presents results obtained by the multiplex SNP marker sets (1) to establish a marker framework in two previously uncharacterized F2 mapping populations, and (2) to determine the genetic relationships between different sugar beet breeding lines. Further applications of the LG-specific SNP multiplex marker sets are discussed.

\section{Materials and methods}

\section{Plant material and DNA}

The plant material was developed by the breeding companies KWS SAAT AG, Einbeck, and STRUBE-
DIECKMANN, Sülbeck. Genomic DNA of three different $\mathrm{F} 2$ populations (K1, K2 and D2), as well as of their parental and F1 genotypes was available. The $\mathrm{K} 2$ population was formerly described as the population 618 by Schneider et al. $(1999,2001,2002)$ and Hunger et al. (2003). The KWS SAAT AG provided also 24 different sugar beet breeding lines.

\section{Generation of PCR primers and singleplex PCR amplification}

STS primers were designed on the basis of sequenced RFLP probes developed by Barzen et al. (1992, 1995) and indicated with the marker IDs MP0002, MP0018, MP0038, MP0057, MP0059, MP0075, MP0081, MP0094, MP0098, MP0111, MP0117, MP0155, MP0167, MP0168, MP0176 in Table 1. All other primers were adopted from Schneider et al. (1999, 2001, 2002) or Hunger et al. (2003). PCR amplification was performed with $50 \mathrm{ng}$ genomic DNA as template in a total volume of $25 \mu$ containing 0.2 $\mathrm{mM}$ dNTPs, $2 \mathrm{mM} \mathrm{MgCl}_{2}, 0.4 \mu \mathrm{M}$ of each primer (Invitrogen, Karlsruhe, Germany) and $0.5 \mathrm{U}$ of Taq DNA polymerase in the reaction buffer provided by the manufacturer (Invitrogen, Karlsruhe, Germany). PCR samples were amplified using the following protocol: initial denaturation for $15 \mathrm{~min}$ at $95^{\circ} \mathrm{C}, 36$ cycles of $1 \mathrm{~min}$ at $94{ }^{\circ} \mathrm{C}, 1 \mathrm{~min}$ at $56-60^{\circ} \mathrm{C}, 2 \mathrm{~min}$ at $72{ }^{\circ} \mathrm{C}$ and a final extension step for $10 \mathrm{~min}$ at $72{ }^{\circ} \mathrm{C}$.

\section{Multiplex PCR amplification}

Five to six different PCR products were amplified in the same reaction volume of $10 \mu \mathrm{l}$ using the Qiagen ${ }^{\circledR}$ Multiplex PCR Kit (Qiagen, Hilden, Germany) with 30 ng genomic DNA as template containing $0.1 \mu \mathrm{M}$ of each primer in the multiplex PCR buffer including dNTPs, $6 \mathrm{mM} \mathrm{MgCl}_{2}$ and HotStartTaq ${ }^{\circledR}$ DNA Polymerase. The amplification conditions were the same as in the singleplex PCR reaction, with the annealing temperature set to $56{ }^{\circ} \mathrm{C}$ in all cases.

\section{Purification and sequencing of PCR products}

Unincorporated dNTPs and primers were removed from the PCR assays using the ExoSAP-IT (Exonuclease I/ Shrimp Alkaline Phosphatase enzyme mixture, USB, Cleveland, Ohio) according to the protocol provided. Single PCR products amplified from the parental lines of the three mapping populations were then sequenced by the MPIZ DNA core facility on 
Table. 1. Characteristics of 52 published sugar beet molecular markers selected for the development of LG-specific multiplex SNP marker sets. Markers are grouped according to their association with LGs. Horizontal lines indicate the distribution of the markers into LGs.

\begin{tabular}{|c|c|c|c|c|c|}
\hline Marker ID & $\begin{array}{l}\text { Marker type; refer- } \\
\text { ence }\end{array}$ & Accession number & Forward primer & Reverse primer & $\begin{array}{l}\text { ampli- } \\
\text { con size } \\
\text { (bp) }\end{array}$ \\
\hline MP0175 & $\boldsymbol{\square} ; \mathrm{b}, \mathrm{d}, \mathrm{e}, \mathrm{f}$ & AY535049 & ATACCACAACTTGCGGTTGC & GGCAATTCTTGAACACGCAG & 1700 \\
\hline & $\mathbf{\Delta} ; \mathrm{f}$ & BG577448 & CGAGGTCTCTATTCACTCAC & CCCAAAACTCCAGCCATAAC & 500 \\
\hline ndk & $\overline{\mathbf{\Delta}} ; \mathrm{d}, \mathrm{e}, \mathrm{f}$ & AF295650 & GTTGTTGCTATGGTCTGGGA & ATGAGTGWAGGCTGCTYTGC & 1600 \\
\hline $\mathrm{nr}$ & $\overline{\boldsymbol{\Delta}} ; \mathrm{c}, \mathrm{d}, \mathrm{e}, \mathrm{f}$ & AF173664 & GATGAACAACTGCTGGTTYCG & TTAACCACDAGATCRAAGTACC & 800 \\
\hline ps2 & $\overline{\mathbf{\Delta}} ; \mathrm{d}, \mathrm{e}, \mathrm{f}$ & BG577449 & AGCAATGCTACTTATGCCTAG & CCAATCATAGCAACACGACC & 480 \\
\hline cab4 & $\Delta ; d, e, f$ & BG577456 & TTACCTTGGCCAACCACTTC & СACTCTACAGGAACTTGTCC & 300 \\
\hline $\mathrm{rbcS}$ & $\overline{\mathbf{\Delta}} ; \mathrm{c}, \mathrm{d}, \mathrm{e}, \mathrm{f}$ & BG577455 & CTCARGCCARCATGGTGGCT & GGCTTGTARGCRATGAAACTGA & 950 \\
\hline invew & $\overline{\boldsymbol{\Delta}} ; \mathrm{c}, \mathrm{d}, \mathrm{e}, \mathrm{f}$ & X81797 & GCAATATGGGGGAACATGAC & CCTCCATAGTCATACCTCCA & 700 \\
\hline сус & $\overrightarrow{\mathbf{\Delta}} ; \mathrm{d}, \mathrm{e}$ & Z71705 & AGAGTACAAACTGGTCCCTG & AGCTGCATGGACATATCTCC & 800 \\
\hline MP0180 & $\overline{\mathbf{D}} ; \mathrm{b}, \mathrm{d}, \mathrm{e}, \mathrm{f}$ & AY535051 & AAAGGCTCCAACTAACCTCC & ACAGGTTCATCGTGCTACAC & 1400 \\
\hline MP0176 & $\bar{\square} ; \mathrm{b}, \mathrm{f}$ & AY535050 & TGGGATGCTTAATTTTCCTTGC & GGGGAAACGACAATCATTCAG & 1400 \\
\hline pmc & $\boldsymbol{\Delta} ; \mathrm{d}, \mathrm{e}, \mathrm{f}$ & BG577451 & AGGAGCTGTCTCTGCATTTG & GTTGTAGGAATGGCTTGTGG & 350 \\
\hline tk & $\overline{\mathbf{\Delta}} ; \mathrm{c}, \mathrm{d}, \mathrm{e}, \mathrm{f}$ & AF173677 & GGTTTTGGSTCTCCTAACAAG & GAGCATMAGAATGTTGGGCAT & 400 \\
\hline MP0044 & $\overline{\mathbf{Z}} ; \mathrm{a}, \mathrm{b}, \mathrm{d}$ & AY535052 & СТTCTAACTGCACTGTGTCC & СТTTTCTGCCCTGACTAACC & 900 \\
\hline MP0167 & $\overline{\mathbf{a}} ; \mathrm{b}$ & AY535053 & GAGAATGTAGGATCAGCGAAG & TGCAGACGTAAACAGTGTG & 1200 \\
\hline MP0111 & $\overline{\mathbf{B}} ; \mathrm{b}$ & AY535054 & TGCTCTTCCTCTTTTGTTTACC & GCCACAGGAAGAACCATAAC & 1200 \\
\hline MP0143 & $\mathbf{\square} ; \mathrm{b}, \mathrm{d}, \mathrm{f}$ & AY535055 & AGCCTGTGATATGCTGCTAG & GAACTCAGGACAGCTATCTG & 1800 \\
\hline MP0132 & $\mathbf{\square} ; \mathrm{b}, \mathrm{d}, \mathrm{e}, \mathrm{f}$ & AY535056 & ACAAGCAGCAAAGTTACACAC & СTTCTTTGCTCAGATTGTATCT & 1700 \\
\hline MP0117 & $\overline{\mathbf{Z}} ; \mathrm{b}, \mathrm{f}$ & AY535057 & GCAGTCATCACATTTCACAATC & AGGATCGACAAATTGATGGAAG & 1900 \\
\hline MP0019 & $\overline{\mathbf{B}} ; \mathrm{b}, \mathrm{d}, \mathrm{e}, \mathrm{f}$ & AY535058 & ATGTAAGTCAAGGCTCCCAG & ССТАCTAACAGCAGGTTACC & 2300 \\
\hline & $\overline{\mathbf{\Delta}} ; \mathrm{c}, \mathrm{d}, \mathrm{e}, \mathrm{f}$ & AY535079 & TGGAGAGGAAACACTGCYAATGT & ATGTTRGCACCAGCWCCCTTGA & 1000 \\
\hline MP0079 & $\overline{\mathbf{D}} ; \mathrm{a}, \mathrm{b}, \mathrm{d}, \mathrm{e}, \mathrm{f}$ & AY535059 & CTGCAGACATTCTCTTGCTC & TTCCACTTTGGGCACCTCAA & 1600 \\
\hline fbpcp & $\mathbf{\Delta} ; \mathrm{d}, \mathrm{e}, \mathrm{f}$ & BG577465 & ATGGCATCAATAGGACCTGC & GCTCGTTGAACTAAGGAAGC & 400 \\
\hline MP0038 & $\overline{\mathbf{a}} ; \mathrm{a}, \mathrm{b}, \mathrm{f}$ & AY535075 & CTTTGTTGCCAATGACTGGC & GTGGTACTGAGTGGTATTCC & 1600 \\
\hline & $\overline{\boldsymbol{\Delta}} ; \mathrm{d}, \mathrm{e}, \mathrm{f}$ & BG577458 & ACTTTGCCTCATCATCGACC & TGTTGTGCCATGGGTCTGAT & 500 \\
\hline & $\overline{\mathbf{\Delta}} ; \mathrm{d}, \mathrm{e}, \mathrm{f}$ & AF295645 & GCTYTKAGRATGTGGGCTTCT & AAACACCACMTCYCCWAGATG & 1000 \\
\hline MP0040 & $\overline{\mathbf{D}} ; \mathrm{a}, \mathrm{b}, \mathrm{d}, \mathrm{e}, \mathrm{f}$ & AY535060 & AGGTTAAGGCTGATGTCTTTG & CTGTGGGAAACACTTACTGG & 1600 \\
\hline aep & $\overline{\mathbf{\Delta}} ; \mathrm{d}, \mathrm{e}, \mathrm{f}$ & AY535077 & GAATCAAGGACGGGAAGTTC & AGCGAGATTGACTGGAGTTG & 1800 \\
\hline oеc & $\Delta ; \mathrm{f}$ & AY535078 & TTCTTACACCACCATGCAGC & GAATCCATCACCGTTGTAGG & 900 \\
\hline sht & $\mathbf{\Delta} ; \mathrm{d}, \mathrm{e}$ & AF295655 & GGACATCTTTCACACGGATAC & TGGATGACATAACTAGCAGGG & 500 \\
\hline pgdh & $\bar{\Delta} ; \mathrm{d}, \mathrm{e}$ & BG577467 & TTGCTGGCCTAGCTGTTATG & TCACCACCTGAAACTCCCAT & 400 \\
\hline MP0094 & $\overline{\mathbf{E}} ; \mathrm{b}, \mathrm{f}$ & AY535061 & AGTCACAGCAAGAGGGGATAAG & TGTGGGGCTGATAGAATCGTC & 1500 \\
\hline MP0168 & ; b & AY535062 & GATTTTGGAGGTGGTGTAGAC & GCTTCTTACCTGTCTTGTCTC & 1500 \\
\hline MP0110 & $\overline{\mathbf{\sigma}} ; \mathrm{b}, \mathrm{d}, \mathrm{e}$ & AY535063 & TTCAAGCAGATATGCCGAAG & AGCTGTCACAAAAAGCAACC & 300 \\
\hline MP0075 & $\mathbf{\square} ; \mathrm{a}, \mathrm{b}, \mathrm{f}$ & AY535064 & ACCTTTATTACAGCCAAGTGCC & ATCTTATACCCAGCCCAGCAAC & 1000 \\
\hline MP0098 & $\overline{\mathbf{B}} ; \mathrm{b}, \mathrm{f}$ & AY535065 & TGTCTTTCCTCTCCTTTTCCC & TCTCTTAAACCAACCAAGTGC & 350 \\
\hline 7M20 & $\overline{\mathbf{O}} ; \mathrm{f}$ & BU089561 & GCTGATCTTCCTAGGTTGG & GCATGAGTAATGCTCTCAGG & 400 \\
\hline 2G14 & ; f & BU089576 & GGTTTGCACTTTTCTTAGATGG & GAGCCAATCAATCTTCAGCC & 400 \\
\hline 6L04 & ; f & BU089564 & ААСССТТСТСТТСАССТСС & CATGAAAGCGAAGCGAAAC & 400 \\
\hline $8 \mathrm{M} 01$ & - ; f & BU089572 & CCCGAGCTTAAGCAACTC & CCCCAATTGTTTTCGTGCC & 400 \\
\hline MP0155 & $\mathbf{\square} ; \mathrm{b}, \mathrm{f}$ & 666 & GCGA & TGATGATGCTGACAGGATCAC & 1200 \\
\hline MP0059 & $\overline{\mathbf{a}} ; \mathrm{a}, \mathrm{b}, \mathrm{f}$ & AY535067 & ACAGTAACGAGGTCAAAGACGC & TGGCTATCGCCGCAACAATC & 800 \\
\hline MP0068 & $\overline{\mathbf{D}} ; \mathrm{a}, \mathrm{b}, \mathrm{d}, \mathrm{e}, \mathrm{f}$ & AY535068 & AGCTTCTCTCTGCTTCAAGC & СТCСАTССТСТАGTTTCTCC & 1400 \\
\hline MP0015 & $\overline{\mathbf{D}} ; \mathrm{a}, \mathrm{b}, \mathrm{d}, \mathrm{e}, \mathrm{f}$ & AY535069 & CTGCTTTCAGAGGCAAGAAG & СТССТСТTСАСААТАТСТTGC & 1300 \\
\hline 4M07 & $\overline{\mathbf{e}} ; \mathrm{f}$ & BU089558 & GGTCGAGGTATACACGATG & CACCTTAGCCCCGTAATCAC & 300 \\
\hline sps & $\mathbf{\Delta} ; \mathrm{c}, \mathrm{d}, \mathrm{e}, \mathrm{f}$ & AY535076 & AGCTGTTATGGAAGGTTCATG & TCGGGTCAGGCCTAGCAA & 300 \\
\hline MP0001 & $\mathbf{\square} ; \mathrm{a}, \mathrm{l}$ & A) & GGAGACCACTTTGAGGAACT & TAACATGGAGTAGCTCCCAC & 800 \\
\hline MP0002 & $\overline{\mathbf{B}} ; \mathrm{a}, \mathrm{b}, \mathrm{f}$ & AY535071 & GCAGCAGTTTTCCTCTACATTC & TCCACTGAGAGACAACTGAAAC & 900 \\
\hline MP0081 & $\overline{\mathbf{a}} ; \mathrm{a}, \mathrm{b}, \mathrm{f}$ & AY535072 & AGCTGGTTTGTTCTCCTTTC & тСТСТСТСТСТСТСТСТСТСТС & 1000 \\
\hline MP0018 & $\overline{\mathbf{D}} ; \mathrm{a}, \mathrm{b}, \mathrm{f}$ & AY535073 & AAGCAAACACAGCATTAGCC & GTATGCAAAGTCCAGACAGAAG & 700 \\
\hline MP0004 & $\overline{\mathbf{D}} ; \mathrm{a}, \mathrm{b}, \mathrm{d}, \mathrm{e}, \mathrm{f}$ & AY535074 & TTCAGCTCTCGGAAGCACAT & ATCCATGCAAGCGTACCTGT & 700 \\
\hline sc & $\overline{\mathbf{\Delta}} ; \mathrm{c}, \mathrm{d}, \mathrm{e}, \mathrm{f}$ & U64902 & CAGCTGGTAGAACATCCGAT & TCCAGCACTCTGAAAGATCC & 500 \\
\hline
\end{tabular}

a: Barzen et al. 1992, b: Barzen et al. 1995, c: Schneider et al. 1999, d: Schneider et al. 2001, e: Schneider et al. 2002, f: Hunger et al. 2003; $\square$ : RFLP marker,

$\mathbf{\Delta}$ : marker developed from expressed gene related to carbohydrate metabolism and yield, $\mathbf{O}$ : marker developed from expressed RGA. 
Applied Biosystems (Weiterstadt, Germany) ABI Prism 377 and 3700 sequencers using Big Dye terminator chemistry. Premixed reagents were from Applied Biosystems. Accession numbers of one reference sequence to each marker locus are listed in Table 1.

\section{Multiplex SNaPshot ${ }^{T M}$ reaction, purification and electrophoretic separation}

The SNaPshot ${ }^{\mathrm{TM}}$ primer extension reaction was performed using $3 \mu \mathrm{l}$ ExoSAP-IT treated multiplex PCR product as template in a total volume of $10 \mu \mathrm{l} \mathrm{con-}$ taining $0.25 \mu \mathrm{M}$ of each primer and $0.5 \mu \mathrm{l}$ of the Ready Reaction Mix including ddNTPs and AmpliTaq®DNA polymerase. The primers were developed according to the instructions provided by Applied Biosystems. Their length varied from 24 to 56 nucleotides (nt) with a difference of $6 \mathrm{nt}$ to each other within the same multiplex. The complementary region in the amplified target locus had a size of 24 to $30 \mathrm{nt}$ (see Table 2). To increase primer length up to $56 \mathrm{nt}$, non-homologous poly (dA) or poly (dGACT) tails were added (see Table 2). Only HPLC purified primers were used for the analysis. The following amplification protocol was applied: 25 cycles of $10 \mathrm{sec}$ at $96^{\circ} \mathrm{C}, 5 \mathrm{sec}$ at $50{ }^{\circ} \mathrm{C}$ and $30 \mathrm{sec}$ at $60^{\circ} \mathrm{C}$. After the extension reaction, the products were purified with 1 U CIP (Calf Intestinal Phosphatase, New England Biolabs, Frankfurt, Germany) for $1 \mathrm{~h}$ at $37^{\circ} \mathrm{C}$, followed by an enzyme deactivation step of 15 min at $75^{\circ} \mathrm{C}$. One $\mu \mathrm{l}$ of the purified samples was loaded in $19 \mu \mathrm{l} \mathrm{LiChrosolv} \mathrm{water} \mathrm{(Merck,} \mathrm{Darmstadt,}$ Germany) mixed with $0,2 \mu \mathrm{l}$ GeneScan-120 LIZ size standard, denatured for $5 \mathrm{~min}$ at $94{ }^{\circ} \mathrm{C}$ and immediately moved to ice. Finally, reaction products were separated by capillary electrophoresis on an ABI Prism 310 sequencer using POP4 polymer at $60{ }^{\circ} \mathrm{C}$ for $16 \mathrm{~min}$.

\section{Data analysis}

Primer design and DNA sequence alignments were performed using the GCG software (Genetics Computer Group, University of Wisconsin at Madison, version 10.2). With the same program, extension primers were tested for possible extendable hairpin structure and dimer formation between primers. Capillary electrophoresis data were recorded at a detection level of E5 50/100 and normalized by the GeneScan analysis software, version 3.1. GeneScan data were analysed with the Genotyper 3.7 NT software. In heterozygous samples, peaks smaller than 10 $\%$ of the peak of the alternative allele were discarded from the analysis. The SNaPshot results of 24 different sugar beet breeding lines, together with parents and F1s of the populations D2, K1 and K2, were analysed with the TREECON software version $1.3 \mathrm{~b}$ (Van de Peer and De Wachter 1994) to determine genetic distances based on marker data using the algorithm of Nei and Li (1979). A phylogenetic tree was constructed employing the UPGMA algorithm. Data were resampled 1000 times providing a bootstrap analysis. Linkage analysis was carried out with JoinMap software version 2.0 (Stam 1993; Stam and Van Ooijen 1995). Eighty-five previously published markers (Schneider et al. 1999, 2002; Hunger et al. 2003), as well as segregation data on 128 unpublished markers (Schneider et al., unpublished), were included for the map construction in the three populations D2, K1 and K2. Linkage groups were drawn using MapChart software version 2.1 (Voorrips 2002) and assigned to chromosomes according to Butterfass (1964) and Schondelmaier and Jung (1997).

\section{Results}

Amplification of selected linkage group-specific marker loci

Starting from a total of 196 available RFLP- (Barzen et al. 1992, 1995) and PCR-based markers derived from ESTs (Schneider et al. 1999, 2002; Hunger et al. 2003), a group of markers was considered for each chromosome, to be eventually converted into a single set of LG-specific SNPs. Criteria to choose the marker loci were (1) their homogenous distribution along the linkage map, (2) a high degree of polymorphism in representative breeding lines, and (3) the possibility to multiplex the markers for PCR amplification and primer extension. For all markers, PCRbased assays were established, and a pre-screen based on SSCP was performed to identify polymorphisms between the parental lines of three mapping populations. Markers showing more than one allele in this panel were selected, and the DNA sequence of alleles of the parental genotypes was determined for these markers. Among these loci, 27 anonymous RFLPs and 25 expressed genes, of which 20 encode gene products potentially affecting carbohydrate metabolism and five show similarities to resistance gene 
Table 2. Details of SNP assays for 52 genetic markers organized in LG-specific multiplexes.

\begin{tabular}{|c|c|c|c|c|}
\hline SNP marker ID & SNP position & Multiplex set ID & Primer sequence $\left(5^{\prime}-3^{\prime}\right)$ adjacent to SNP position & Primer orientation* \\
\hline MP0175 & 290 & AMI & GTCACACGGAATGATATCTGATGAAATTGG & $5 '-3$, \\
\hline MPatp3 & 314 & AMI & GATCGATCGATCGATCCCAGAATTGGTGATGAAATC & $5,-3$, \\
\hline MP_ndk & 344 & AMI & AAAAAATATCCCTTGTGTTTCCGCATTTTATCGTATTAACTC & $3 '-5$ ' \\
\hline MP_nr & 280 & AMI & $\begin{array}{l}\text { GATCGATCGATCAGCCTGGATTGTTGT- } \\
\text { TCATGGACATGTGTATGATGC }\end{array}$ & $5 '-3{ }^{\prime}$ \\
\hline MP_ps2 & 259 & AMI & $\begin{array}{l}\text { GACTGACTGACTGACTGTACTAACCT- } \\
\text { TCTTGACAGGAGCTTTGGTTTTGGGCTT }\end{array}$ & $3^{\prime}-5$ \\
\hline MPcab4 & 296 & AMII & TGAATTCCAGAGGAATGCAGAGCT & $5 '-3$ ' \\
\hline MPrbcS & 156 & AMII & CCTGTCACCAGGAAAAGCAATGACATTACT & $5 '-3$, \\
\hline MPinvew & 172 & AMII & AAAAAATTGCTTCAGTGGGTCAATCACAATGCTTCC & $5 '-3{ }^{\prime}$ \\
\hline MP_cyc & 135 & AMII & GACTGACTGACTAACTTGGGGGGCACTCATTTCTTCGTACTT & $3^{\prime}-5$ ' \\
\hline MP0180 & 371 & AMII & $\begin{array}{l}\text { CTGACTGACTGACTGACTGCAGCAAG- } \\
\text { GATGAACAACAATAGAGACATC }\end{array}$ & $5^{\prime}-3$ \\
\hline MP0176 & 171 & AMII & $\begin{array}{l}\text { TCGATCGATCGATCGATCGTTTCT- } \\
\text { GATATTTCCATGTGAAATCAACAAGTAGAG }\end{array}$ & $5 '-3$ ' \\
\hline MP_pmc & 200 & AMIII & AAGTTTCTCCСTATCAGCTCTGTA & $3^{\prime}-5$ ' \\
\hline MP_tk & 143 & AMIII & ACGTTCCAGAGGAAGTTAAGAAGTAAGCAA & $5 '-3$ ' \\
\hline MP $\overline{00} 44$ & 555 & AMIII & AAGATGGGCCTTTCCAGAGATGAATTTTTCCACAGA & $3^{\prime}-5$ ' \\
\hline MP0167 & 544 & AMIII & $\begin{array}{l}\text { CTGACTGACTGACTGACTCTAGTGACT- } \\
\text { GACATCACCCTACTAAAAGTA }\end{array}$ & $3^{\prime}-5$ ' \\
\hline MP0111 & 436 & AMIII & $\begin{array}{l}\text { GACTGACTGACTGACTTGATCAA- } \\
\text { GATAAGGATAGAGCACAAACCAAGCTAAT }\end{array}$ & $3{ }^{\prime}-5$ \\
\hline MP0143 & 159 & AMIV & TGCGTGTGCAAAGATTCAGAGTCA & $3^{\prime}-5$ ' \\
\hline MP0132 & 368 & AMIV & ATGGCATCAGTAACGGATGGACTGTATTTA & $3^{\prime}-5$, \\
\hline MP0117 & 352 & AMIV & ССTGCTTGTTACTTTACTCTCACATTTTAGTCTCGG & $3^{\prime}-5$ ' \\
\hline MP0019 & 150 & AMIV & AAAAAATGTATCTGAAAACCAGGATGCATGGGGAAATATTGC & $5,-3$, \\
\hline MP_ant & 489 & AMIV & $\begin{array}{l}\text { GACTGACTGACTAAACCACTTCCAG- } \\
\text { TAACCATCTCTGTCCTTCTTGAA }\end{array}$ & $3{ }^{\prime}-5$ \\
\hline MP0079 & 349 & AMIV & $\begin{array}{l}\text { CTGACTGACTGACTGACTAATGTAGGAT- } \\
\text { GAGTGGAAGTAAAAGAAATGGAGGGG }\end{array}$ & $3{ }^{\prime}-5$ \\
\hline MPfbpcp & 224 & AMV & CAAAGTCTCTTCACTCATTTCCCT & $5,-3$, \\
\hline MP0038 & 117 & AMV & ATCTACCAAAATGCCAAGGTCTGCCTCCTT & $5 '-3$ ' \\
\hline MPcab5 & 429 & AMV & AAAAAAAAGGAGAAGGAGCTTGCTAATGGTATGCCT & $5 '-3$ ' \\
\hline MP_gdh & 128 & AMV & AAAAAAAGCTCTGTGGAATAATATATCTTTCGCCCAACCAAA & $3 '-5$ ' \\
\hline MP0040 & 487 & AMV & $\begin{array}{l}\text { GACTGACTGACTTTCAACAAGCT- } \\
\text { GAAAAATATCAGCAAGACGAACTTC }\end{array}$ & $5 '-3$ ' \\
\hline MP_aep & 211 & AMV & $\begin{array}{l}\text { CTGACTGACTGACTGACTTCTTCTGAT- } \\
\text { GCTGTTTACTAATGAGTCATTTGACAA }\end{array}$ & $5^{\prime}-3$ \\
\hline MP_oec & 263 & AMVI & ATTTGGTATCACTGTGAGTTTCCA & $5,-3$, \\
\hline MP_sht & 281 & AMVI & AGCAAGGAGCAATTATGTTCAATGCTAGGC & $5,-3$, \\
\hline MPpgdh & 466 & AMVI & AATCCAGAAACCTCGTGTTATAATCATGCTTGTTAA & $5,-3$, \\
\hline MP0094 & 631 & AMVI & AAAAAATCTTCTGCCCCAATAGAGGAGCTAAAAACAATCTAG & $5,-3$, \\
\hline MP0168 & 885 & AMVI & $\begin{array}{l}\text { GACTGACTGACTAGTCATCTTGACAGCT- } \\
\text { TCAAGGAAGCCCTTTTGACT }\end{array}$ & $5^{\prime}-3$ \\
\hline MP0110 & 475 & AMVI & $\begin{array}{l}\text { GACTGACTGACTGACTGACTGACTGG- } \\
\text { GAGATCCACAGTTAATTTTTTACTTCAA }\end{array}$ & $5 '-3$ ' \\
\hline MP0075 & 785 & AMVII & GTTTTAAGGGATTGAAGGGTGGCA & $5,-3$, \\
\hline MP0098 & 352 & AMVII & ATAGAGCACCAATTTTAGCAATGCAGTGCA & $5,-3$ \\
\hline MP7M20 & 1249 & AMVII & TGAGCTTCCTCTTTTTCTCTCTAATTGTTCATCACT & $5,-3$, \\
\hline MP2G14 & 438 & AMVII & AAAAAAAAAAAAAGTAACATAGGCATGTCTCCAAGAGAGGGT & $3 '-5$ ' \\
\hline MP6L04 & 527 & AMVII & $\begin{array}{l}\text { CTGACTGACTGACTGACTACAGAGGTTC- } \\
\text { CAAATGGAAGGGGAGAGAGA }\end{array}$ & $3^{\prime}-5$ ' \\
\hline MP8M01 & 1136 & AMVII & $\begin{array}{l}\text { AAAAAAAAAAAAAATTGTTTTCGTGC- } \\
\text { CAGTGAATGCCGAATATGAATGGAAGAT }\end{array}$ & $3^{\prime}-5$ ' \\
\hline MP0155 & 529 & AMVIII & CTTCCTTCCGGCAATGGAATCTAA & $3^{\prime}-5$, \\
\hline MP0059 & 617 & AMVIII & CCCAGAAGTTTCAAAGTGCATGCCCAGAAA & $5,-3$, \\
\hline MP0068 & 346 & AMVIII & GACTGACTGACTTGCAGTGTTCATGTCTAGATATTT & $5,-3$, \\
\hline MP0015 & 335 & AMVIII & GACTGACTGACTACATAATGATCAAGGCTTCCATGCTCTAAC & $5,-3$, \\
\hline MP4M07 & 678 & AMVIII & $\begin{array}{l}\text { GACTGACTGACTACCCAAACTAC- } \\
\text { CATTTGGCATATACTCATAAACAAG }\end{array}$ & $3^{\prime}-5$ ' \\
\hline
\end{tabular}


Table 2. Continued.

\begin{tabular}{|c|c|c|c|c|}
\hline SNP marker ID & SNP position & Multiplex set ID & Primer sequence $\left(5^{\prime}-3^{\prime}\right)$ adjacent to SNP position & Primer orientation* \\
\hline MP_sps & 332 & AMVIII & $\begin{array}{l}\text { CTGACTGACTGACTGACTGTACCAG- } \\
\text { TATCTACCAAATGACCCACCAGATAGCTT }\end{array}$ & $3{ }^{\prime}-5$ \\
\hline MP0001 & 429 & AMIX & TAAGTCAGCAACTTAACACACACA & $3^{\prime}-5$ \\
\hline MP0002 & 720 & AMIX & TAAGACTTTTGGTTTGGATTTAGACTCAAG & $3 '-5$ ' \\
\hline MP0081 & 605 & AMIX & GACTGACTGACTGGTGATATCATGTCCTATTCCTAT & $5,-3$ ' \\
\hline MP0018 & 503 & AMIX & AAAAAAATGGTATGCAAGCTCAACAAGTGGAAGGAATAGGTA & $5^{\prime}-3^{\prime}$ \\
\hline MP0004 & 345 & AMIX & $\begin{array}{l}\text { CTGACTGACTGACTGACTCTGCTTTGAT- } \\
\text { TCTTCAAGTTGGTTCATCAG }\end{array}$ & $3{ }^{\prime}-5$ \\
\hline MP_sc & 753 & AMIX & $\begin{array}{l}\text { GACTGACTGACTGACTGACTGACTGCT- } \\
\text { TGGAGATGCTAAGAAGGTGCTTAATCG }\end{array}$ & $5^{\prime}-3$ \\
\hline
\end{tabular}

* 5'-3': primer sequence on sense strand of reference sequence, see accession numbers in Table 1; 3'-5': primer sequence complementary to reference sequence.

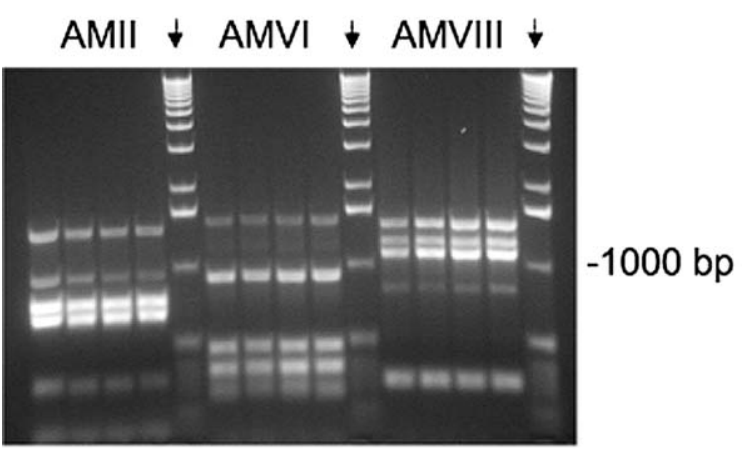

Figure 1. Multiplex PCR of LG-specific marker sets; Ethidium bromide-stained agarose gel revealing the amplification products of multiplex sets AMII, AMVI and AMVIII for four genotypes of the D2 population. Arrows indicate lanes with molecular size markers (1kb ladder).

analogues (RGAs), were finally assembled into the LG-specific multiplex marker sets. Table 1 summarizes the details on the marker sets, in which for each linkage group from five to six SNP marker loci are available. References to the source of linkage data are given for each specific marker; accession numbers of the DNA sequence representing the chosen loci are listed, and information concerning amplification primers and length of genomic PCR products is provided.

Multiplex assays were optimized to allow the simultaneous amplification of the complete set comprising five to six marker loci per LG in a single reaction. Successful amplification was monitored for random samples in agarose gels. An example of this experimental part is reported in Figure 1 revealing the multiplex PCR products for three linkage groups in four genotypes each. Discrete bands are recognized in each lane corresponding to locus-specific PCRamplified products. The experiment was considered positive when the predicted number of amplified products with the correct size was actually present for each marker set and when amplicons could be distinguished according to their size. The procedure which has been developed allows to carry out this preliminar PCR amplification reaction simultaneously for six loci of linkage groups 2, 4, 5, 6, 7, 8 and 9 and for five loci of linkage groups 1 and 3. Differences between the same band present in different genotypes, reflecting different alleles, were predicted to be found and used for SNP analysis.

\section{Development of primers and conditions for multiplexing LG-specific SNP assays}

The amplification products described in the previous section represented the basis for a further step of amplification involving the sequence-specific elongation of a primer adjacent to an SNP position. The technique chosen to reveal SNP alleles is based on primer extension by fluorescently labelled nucleotides (SNaPshot $^{\mathrm{TM}}$, Applied Biosystems, Foster City, CA, USA; see also material and methods). Table 2 reports details necessary to characterize the 52 genetic marker loci revealed in the frame of this SNP analysis. Similar to what was done to amplify the selected marker loci, also the single nucleotide extension was multiplexed starting from the pool of PCR products representing a set of linkage group-specific loci. A good resolution of fluorescent primer extension products by capillary electrophoresis was considered important. To this end, oligonucleotides created to reveal 
SNP reactions had lengths varying between 24 to 56 nucleotides.

A further problem, which specifically affects multiplex assays, concerns primer dimer formation. Therefore, care was taken that all the extension primers of one multiplex set had no long homologous regions that could result in dimer formation. Thirty of the 52 SNP primers showed no putative hairpin structure according to secondary structure analysis. The remaining 22 extension primers allowed one to three possible stem loop formations with a minimal stem length of $6 \mathrm{bp}$ and a loop size between three and 20 nucleotides. In these cases, no other primers were found to avoid potential secondary structure formation.

After primer extension, the labelled SNP primers were separated by capillary electrophoresis according to their size. The position, quality and quantity of the fluorescent signals were recorded by a laser beam and processed by genotyper software to produce genotyping results in the form of the example given in Figure 2.

To compensate weak signals, as initially recorded for the alleles of the markers MP0038 and MP0059, the peak detection level was lowered to E5 20/50. Under these conditions, genotyping results could be reliably evaluated.

All SNaPshot primers of the nine LG-specific multiplex sets produced results in the analyses of 30 sugar beet breeding lines with the exception of MP0044, MP_oec and MP0068 (Table 3). These primers were, however, successfully used in genotyping F2 populations (Figure 3). On the other side, for the six SNP loci MP0167, MP0132, MP0117, MP0019, MP_aep and MP0015, genotyping in F2 populations failed repeatedly, and segregation data obtained by other mapping techniques had to be substituted in these cases (Figure 3). Although PCR products were present, no or only very weak fluorescence signals were obtained after capillary electrophoresis for MP0132, MP0117 and MP0015. In these cases, lowering the detection level to E5 20/50 had no effect on peak detection. Four of the nine primers failing reliable SNP scoring showed one possible hairpin structure: MP0068, MP0132, MP0019 and MP_aep. Nevertheless, all SNP assays produced results in either the segregation analyses or in the DNA fingerprinting experiment; some primers with predicted hairpin structures were even successfully used in both experiments. We conclude that under the conditions

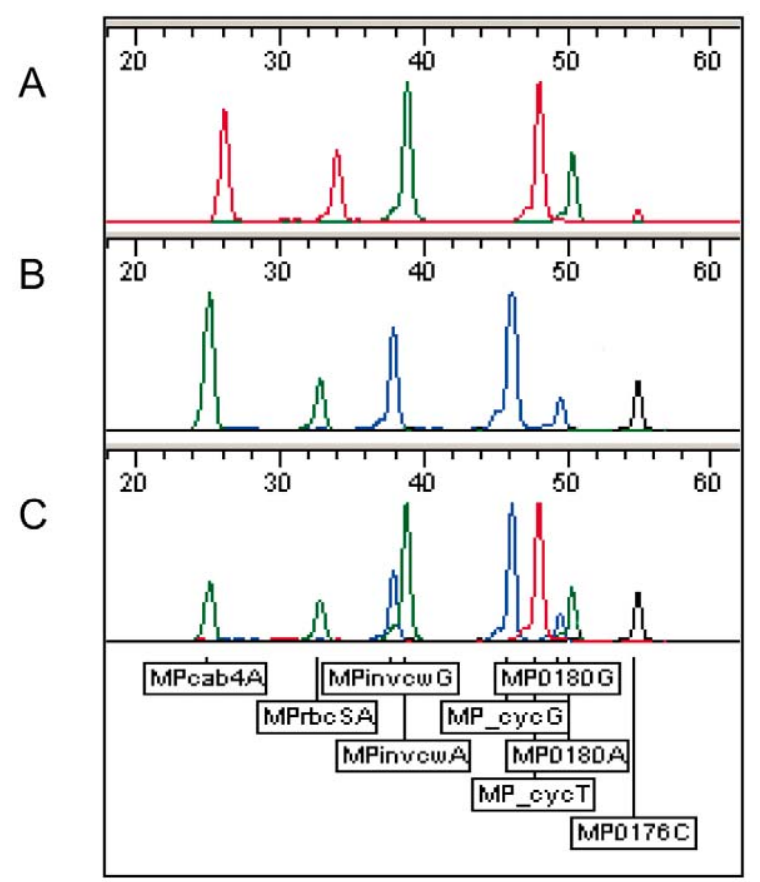

Figure 2. Multiplex SNP scoring using the SNaPshot method based on four different fluorescently labelled nucleotides and genotyper software for data analysis; The diagram shows peak positions and sizes for the alleles of the six SNP markers of multiplex AMII in three genotypes (A: K1P1, B: K1P2, C: individual F2 plant heterozygous at the loci MPinvew, MP_cyc and MP0180 and homozygous at the other loci MPcab4, MPrbcS and MP0176; black: C, red: T, green: A, blue: G; see online figure for colour display). The horizontal scale indicates the size of elongated SNP primers in nucleotides. Below panel $\mathrm{C}$, marker alleles are shown in boxes.

chosen, the formation of secondary structures is not a predictive reason for unreliable SNP detection results.

Accuracy of the SNaPshot method was scrutinized by comparing the results of the $\mathrm{K} 1$ and $\mathrm{K} 2$ population to the already known segregation data obtained by other techniques like SSCP. Data of seven markers were verified in population $\mathrm{K} 1$, and 32 in population K2. The segregation data of four markers showed a deviation between 5 to $8 \%$ from the predicted one. In general, the comparison revealed a high accuracy of most of the 39 segregation analyses, with an average error rate of lower than $5 \%$.

\section{Anchoring the 52 SNP loci to three molecular linkage maps}

The linkage map K2 is based on $108 \mathrm{~F} 2$ plants which were also available to this study (Schneider et al. 
Table 3. Characterization of 52 SNP markers organized in multiplex assays. SNP alleles are listed. Allele frequencies at 49 SNP marker loci evaluated in 30 diploid sugar beet breeding lines and the corresponding PIC-values are given.

\begin{tabular}{|c|c|c|c|c|c|c|c|c|}
\hline \multirow{2}{*}{$\begin{array}{l}\text { SNP } \\
\text { marker ID }\end{array}$} & \multirow{2}{*}{$\begin{array}{l}\text { Multiplex } \\
\text { set ID }\end{array}$} & \multicolumn{2}{|c|}{ Allel A } & \multicolumn{2}{|c|}{ Allel B } & \multicolumn{2}{|c|}{ Allel C } & \multirow{2}{*}{$\begin{array}{l}\text { PIC-value } \\
1-\Sigma p_{i}^{2}\end{array}$} \\
\hline & & base & frequency $(p)$ & base & frequency $(p)$ & base & frequency (p) & \\
\hline MP0175 & AMI & $\mathrm{C}$ & 0.84 & $\mathrm{~T}$ & 0.16 & - & - & 0.27 \\
\hline MPatp3 & AMI & A & 0.62 & $\mathrm{G}$ & 0.38 & - & - & 0.47 \\
\hline MP_ndk & AMI & G & 0.88 & A & 0.12 & - & - & 0.21 \\
\hline MP_nr & AMI & $\mathrm{C}$ & 0.50 & A & 0.50 & - & - & 0.50 \\
\hline MP_ps2 & AMI & G & 0.52 & A & 0.48 & - & - & 0.50 \\
\hline MPcab4 & AMII & A & 0.82 & $\mathrm{~T}$ & 0.10 & $\mathrm{G}$ & 0.08 & 0.31 \\
\hline MPrbcS & AMII & A & 0.83 & $\mathrm{~T}$ & 0.17 & - & - & 0.28 \\
\hline MPinvew & AMII & A & 0.83 & G & 0.17 & - & - & 0.28 \\
\hline MP_cyc & AMII & G & 0.63 & $\mathrm{~T}$ & 0.37 & - & - & 0.47 \\
\hline MP0180 & AMII & G & 0.67 & A & 0.33 & - & - & 0.44 \\
\hline MP0176 & AMII & $\mathrm{C}$ & 0.78 & $\mathrm{~T}$ & 0.22 & - & - & 0.34 \\
\hline MP_pmc & AMIII & A & 0.90 & G & 0.10 & - & - & 0.18 \\
\hline MP_tk & AMIII & $\mathrm{C}$ & 0.85 & $\mathrm{~T}$ & 0.15 & - & - & 0.26 \\
\hline MP0044 & AMIII & G & - & A & - & - & - & - \\
\hline MP0167 & AMIII & G & 0.68 & $\mathrm{C}$ & 0.32 & - & - & 0.44 \\
\hline MP0111 & AMIII & $\mathrm{T}$ & 0.65 & $\mathrm{C}$ & 0.35 & - & - & 0.46 \\
\hline MP0143 & AMIV & $\mathrm{C}$ & 0.51 & G & 0.47 & $\mathrm{~T}$ & 0.02 & 0.52 \\
\hline MP0132 & AMIV & A & 0.61 & $\mathrm{~T}$ & 0.39 & - & - & 0.48 \\
\hline MP0117 & AMIV & G & 0.68 & A & 0.32 & - & - & 0.44 \\
\hline MP0019 & AMIV & $\mathrm{G}$ & 0.65 & A & 0.35 & - & - & 0.46 \\
\hline MP_ant & AMIV & $\mathrm{G}$ & 0.75 & A & 0.25 & - & - & 0.38 \\
\hline MP0079 & AMIV & $\mathrm{G}$ & 0.81 & $\mathrm{~T}$ & 0.19 & - & - & 0.31 \\
\hline MPfbpcp & AMV & $\mathrm{C}$ & 0.68 & A & 0.32 & - & - & 0.44 \\
\hline MP0038 & AMV & $\mathrm{C}$ & 0.57 & $\mathrm{~T}$ & 0.43 & - & - & 0.49 \\
\hline MPcab5 & AMV & $\mathrm{C}$ & 0.70 & $\mathrm{~T}$ & 0.30 & - & - & 0.42 \\
\hline MP_gdh & AMV & $\mathrm{C}$ & 0.72 & $\mathrm{~T}$ & 0.28 & - & - & 0.40 \\
\hline MP0040 & AMV & A & 0.59 & $\mathrm{C}$ & 0.41 & - & - & 0.48 \\
\hline MP_aep & AMV & $\mathrm{T}$ & 0.86 & A & 0.14 & - & - & 0.24 \\
\hline MP_oec & AMVI & G & - & $\mathrm{C}$ & - & - & - & - \\
\hline MP_sht & AMVI & A & 0.52 & $\mathrm{G}$ & 0.48 & - & - & 0.50 \\
\hline MPpgdh & AMVI & A & 0.77 & $\mathrm{G}$ & 0.23 & - & - & 0.35 \\
\hline MP0094 & AMVI & A & 0.88 & $\mathrm{C}$ & 0.12 & - & - & 0.21 \\
\hline MP0168 & AMVI & G & 0.75 & $\mathrm{~T}$ & 0.25 & - & - & 0.38 \\
\hline MP0110 & AMVI & $\mathrm{T}$ & 0.76 & C & 0.24 & - & - & 0.36 \\
\hline MP0075 & AMVII & $\mathrm{G}$ & 0.86 & A & 0.14 & - & - & 0.24 \\
\hline MP0098 & AMVII & $\mathrm{T}$ & 0.78 & $\mathrm{G}$ & 0.22 & - & - & 0.34 \\
\hline MP7M20 & AMVII & C & 0.62 & $\mathrm{~T}$ & 0.38 & - & - & 0.47 \\
\hline MP2G14 & AMVII & C & 0.60 & $\mathrm{~T}$ & 0.40 & - & - & 0.48 \\
\hline MP6L04 & AMVII & G & 0.58 & A & 0.42 & - & - & 0.49 \\
\hline MP8M01 & AMVII & G & 0.60 & A & 0.40 & - & - & 0.48 \\
\hline MP0155 & AMVIII & A & 0.88 & G & 0.12 & - & - & 0.21 \\
\hline MP0059 & AMVIII & A & 0.91 & G & 0.09 & - & - & 0.16 \\
\hline MP0068 & AMVIII & A & - & C & - & - & - & - \\
\hline MP0015 & AMVIII & $\mathrm{C}$ & 0.78 & A & 0.22 & - & - & 0.34 \\
\hline MP4M07 & AMVIII & $\mathrm{T}$ & 0.76 & $\mathrm{C}$ & 0.24 & - & - & 0.36 \\
\hline MP_sps & AMVIII & G & 0.57 & A & 0.43 & - & - & 0.49 \\
\hline MP0001 & AMIX & A & 0.72 & $\mathrm{~T}$ & 0.28 & - & - & 0.40 \\
\hline MP0002 & AMIX & $\mathrm{C}$ & 0.52 & $\mathrm{~T}$ & 0.48 & - & - & 0.50 \\
\hline MP0081 & AMIX & A & 0.62 & G & 0.38 & - & - & 0.47 \\
\hline MP0018 & AMIX & $\mathrm{G}$ & 0.83 & C & 0.17 & - & - & 0.28 \\
\hline MP0004 & AMIX & A & 0.55 & $\mathrm{~T}$ & 0.45 & - & - & 0.50 \\
\hline MP_sc & AMIX & C & 0.56 & A & 0.33 & $\mathrm{~T}$ & 0.11 & 0.57 \\
\hline Mean & & & 0.706 & & 0.29 & & 0.004 & 0.39 \\
\hline
\end{tabular}


K2-I
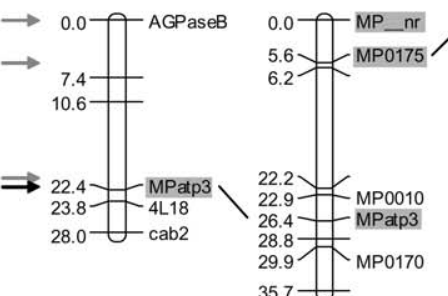

K2-III

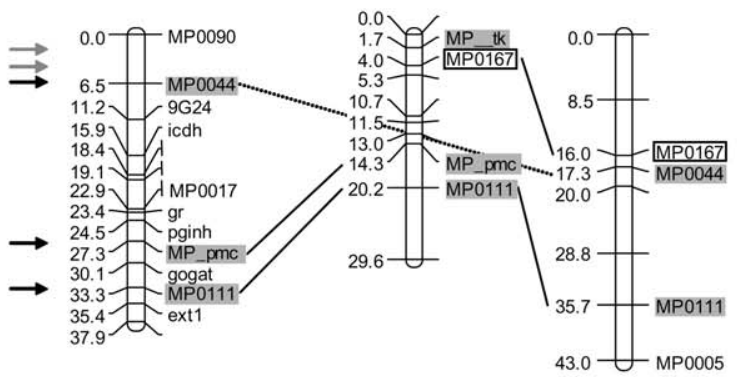

K2-V

K1-V

D2-V
D2-I

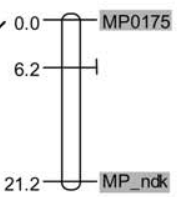

K1-III

D2-III

$3.0-$ MP0005

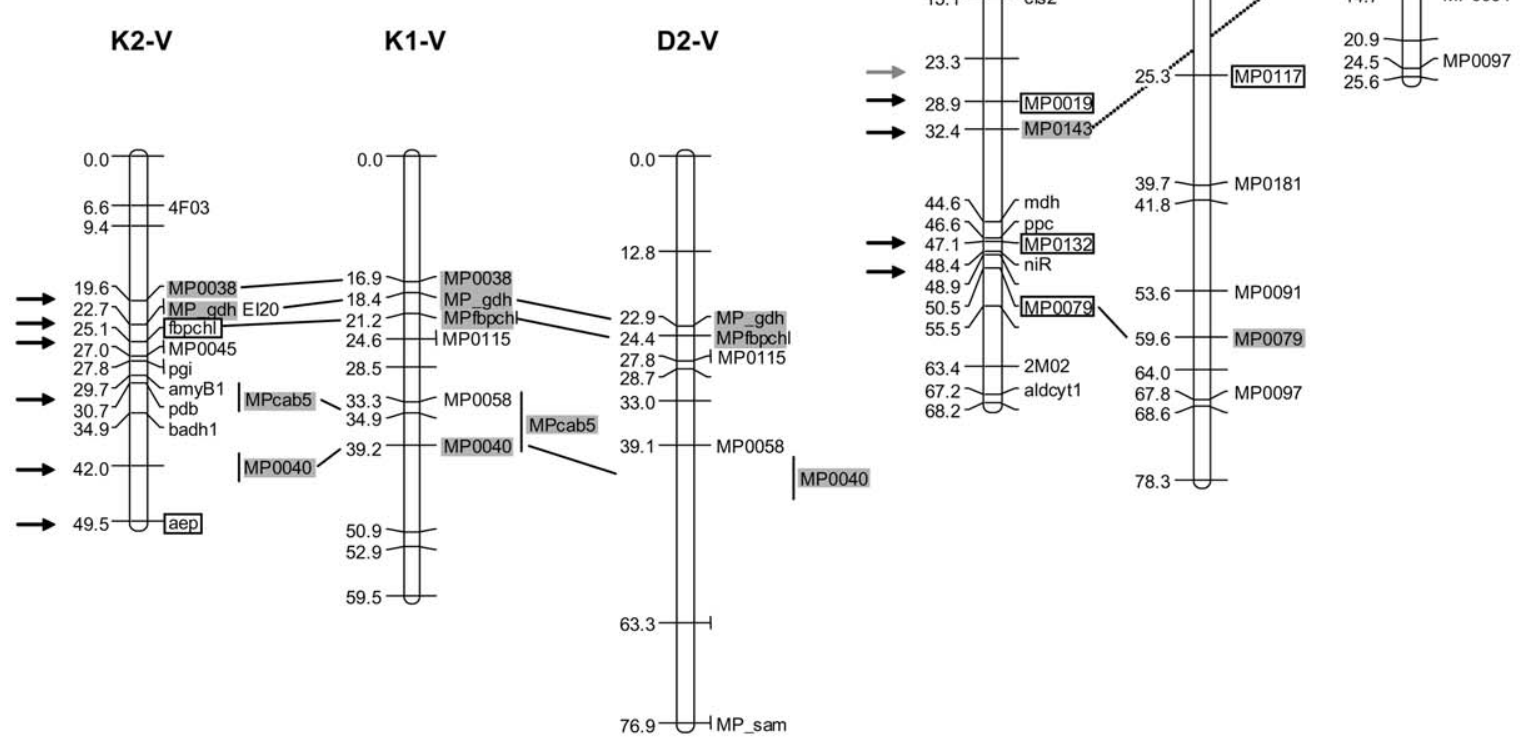

K2-II

K1-II

D2-II

Figure 3. Linkage maps of the three different F2 populations K1, K2 and D2, reporting the positions of SNP anchor markers from the multiplex sets; Marker positions obtained in the second round of Joinmap are included in the map. The most likely positions of markers only analysable in the third round of Joinmap are indicated by vertical bars. SNP markers from multiplex sets are labelled, their positions in the different maps are connected by either straight (K2-K1; K1-D2) or dotted (K2-D2) lines. In those cases, in which the segregation data of these loci were obtained by SNaPshot, these are marked by grey background. In all other cases, the marker symbols are in boxes. The positions of anchor markers are indicated by horizontal arrows to the left of the K2 LGs; black arrows refer to markers mapped directly in $\mathrm{K} 2$, and grey arrows point to the most likely position inferred from other populations. In addition to the LG-specific SNP markers, 85 known loci were assigned to the three maps. The positions of further 128 loci are indicated, but not displayed. 

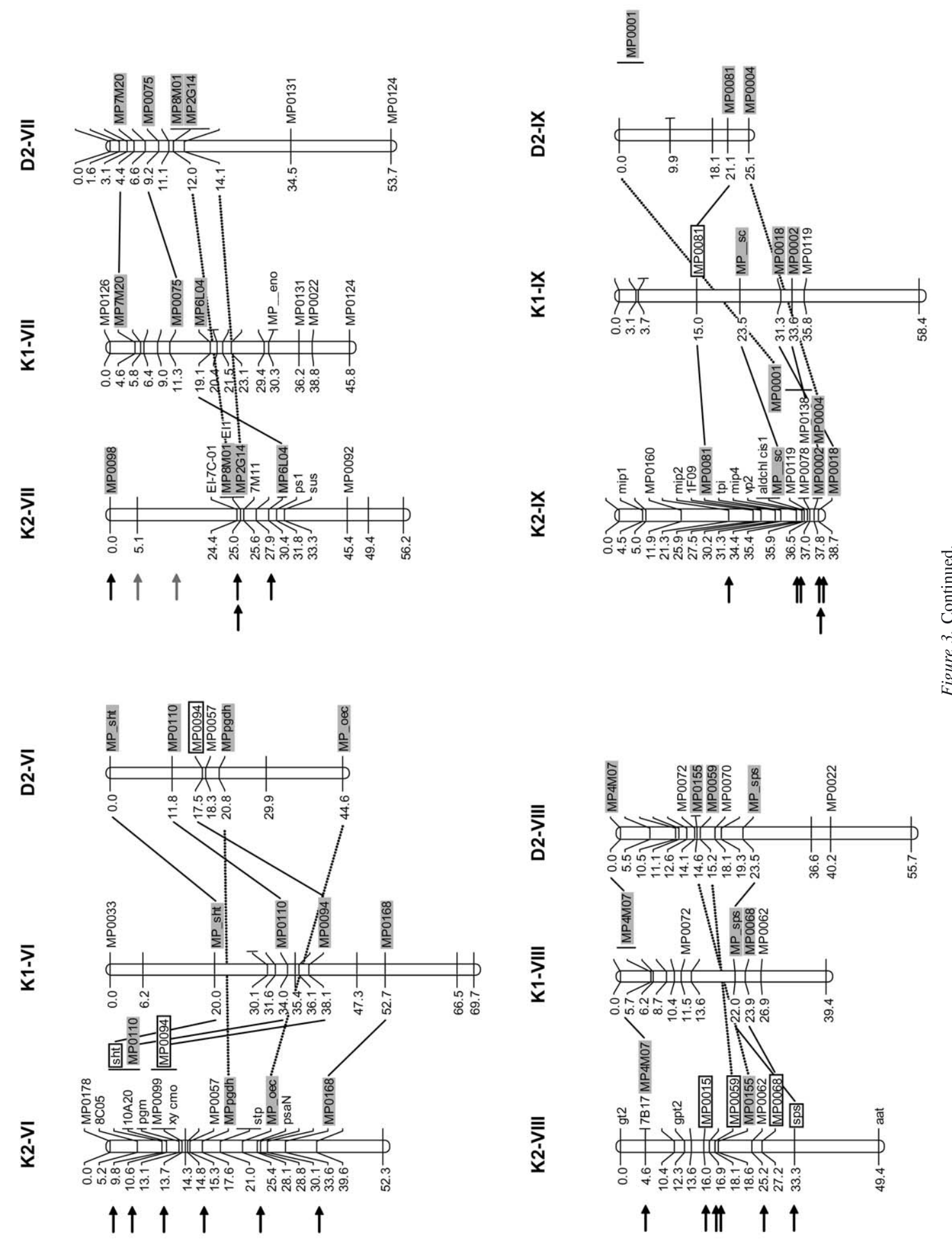
2002; Hunger et al. 2003). The nine sets representing the linkage groups of sugar beet were also evaluated in two further mapping populations which include segregation data of known and yet unpublished loci to support the marker framework (Schneider et al., unpublished). Figure 3 reports positions of the 52 SNP marker loci in the new populations. Relative positions of markers are maintained in the two new maps compared to K2.

Out of the five to six SNP LG-specific markers, 3.8 markers segregated on average simultaneously in the same mapping population. This proved sufficient to identify unambiguously all nine linkage groups in the two new populations. Additionally, due to the presence of two to six segregating markers for 17 out of 18 linkage groups of the two new maps, the orientation of the linkage group with respect to the marker order was possible. Table 3 summarizes the information on the allelic state at the 52 marker loci considered. With three exceptions, only two alleles were found to be present at each marker locus. The three exceptions revealed the presence of three alleles. In Table 3, allele A has been always assigned to the most frequent genetic variant.

\section{DNA fingerprinting of sugar beet breeding lines using the multiplex SNP marker sets}

In the previous section, it was shown that the nine sets of SNP markers help in LG mapping. Not all of the SNP loci present in the sets, of course, were supposed to be polymorphic in all crosses among sugar beet lines. Because of this, their mapping potential was lower than $100 \%$, with $100 \%$ corresponding to the mapping of all 52 marker loci. To estimate the degree of polymorphism of the SNP-based marker sets, allelic frequencies for 49 out of 52 markers were determined in 30 different sugar beet genotypes. In addition, the polymorphism information content values, PIC (Anderson et al. 1993), were calculated (Table 3). With rare exceptions, one of the SNP alleles out of those revealed for each locus prevailed largely on the others. On average, the most abundant allele displayed a frequency of more than $70 \%$, followed by the second most frequent allele occurring in $29 \%$ of all cases and, in rare cases, by a third.

The calculated PIC-values ranged from 0.16 to 0.57 , with an average value of 0.39 . Considering a population of individuals, these values correspond to the heterozygosity index of Nei (1987) which is based on the same algorithm (Schneider et al. 2001). The average value of this index describes, accordingly, the average proportion of heterozygous individuals per locus in a panmictic population as well as the expected proportion of heterozygous loci in a randomly chosen individual. In a random sample of inbred lines, the index describes the proportion of crosses among the same lines which will be heterozygous at a given locus.

The same set of SNP data concerning 30 sugar beet genotypes was analysed by a tree building method based on genetic distances calculated from SNP marker data transformed into a 0/1 matrix (Van de Peer and De Wachter 1994). The phylogenetic tree of Figure 4 indicates that the anchor sets of SNP markers, although monitoring only five to six genetic loci per chromosome, were capable to differentiate all 30 genotypes from each other, including the lines K1P1, $\mathrm{K} 1 \mathrm{P} 2, \mathrm{~K} 2 \mathrm{P} 1, \mathrm{~K} 2 \mathrm{P} 2$ and $\mathrm{D} 2 \mathrm{P} 2$ used to construct the populations $\mathrm{K} 1, \mathrm{~K} 2$ and $\mathrm{D} 2$.

\section{Discussion}

\section{Development of multiplex SNP marker sets}

Multiplex LG-specific SNP marker sets were developed and evaluated in LG-mapping and fingerprinting. The procedure involves (1) the simultaneous PCR amplification of five to six target loci and (2) the simultaneous detection of SNP alleles using capillary electrophoresis and fluorescence measurements. The procedure exceeds the $\mathrm{SNaPshot}$ protocol of Makridakis and Reichardt (2001), which describes initial amplification and primer extension for a maximum of four SNPs simultaneously. It is also more efficient than the protocol of Törjék et al. (2003), in which multiplexing is limited to the primer extension step. Our semiautomated procedure minimizes the number of pipetting steps and therewith the risk of human error. It provides the basis for accurate genotyping in high throughput using multiple capillaries in parallel.

\section{Versatility of multiplexed SNP marker systems}

Previously, mapping systems such as multiple marker lines with visible phenotypes, as known from Arabidopsis thaliana (Koornneef et al. 1987), were used to assess the genotypes at a group of markers in a single step. However, working with multiple marker lines involves the construction of plant populations which 


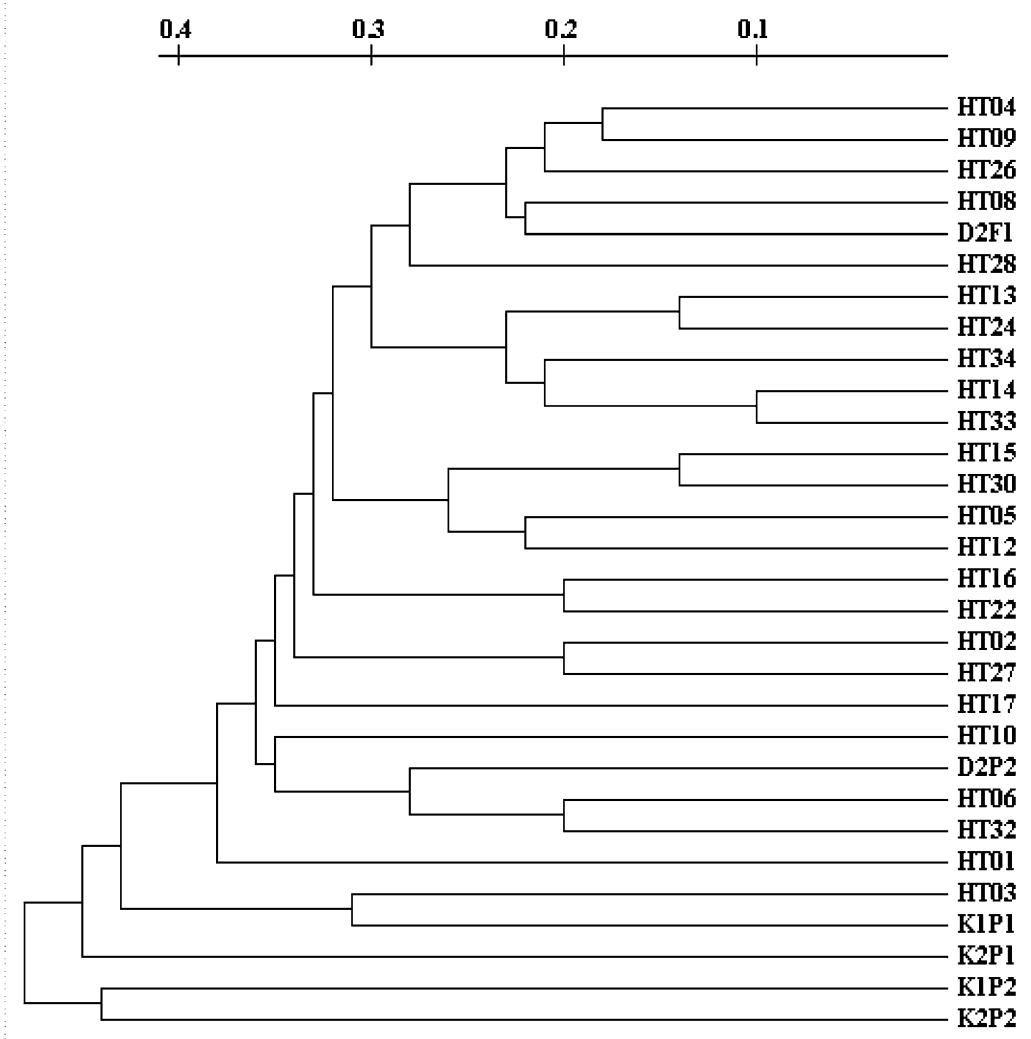

Figure 4. Phylogenetic tree reporting the genetic relationships among 30 sugar beet breeding lines based on 49 SNP markers; The panel includes parental lines of the mapping populations K1, K2, D2 (K1P1, K1P2, K2P1, K2P2 and D2P2), the F1 line of population D2 and 24 further independent breeding lines designated with the prefix HT.

is labour intensive and time consuming. At the molecular level, multiplexed simple sequence repeat (SSR) markers have already been developed for a number of crops such as rape (Mitchell et al. 1997), soybean (Narvel et al. 2000), cotton (Liu et al. 2000) and sunflower (Tang et al. 2003). SSR markers are well established in forensic, clinical, animal and plant research to scan the genome at known loci. They are very polymorphic having numerous alleles per microsatellite locus. In sunflower, the minimum, average and maximum heterozygosity of the multiplexed markers were $0.38,0.62$ and 0.83 , respectively. However, the development of SSR markers is a lengthy process. Additionally, it was shown that SNPs are more abundant than SSRs, thus enabling the generation of a molecular marker at nearly any given site of the genome. SNP markers can not only be analysed electrophoretically, but also in a hybridization-based format using oligo chips (reviewed in Syvanen 2001). This enables the multiparallel analysis of a virtually unlimited number of loci, whereas multiplexing SSR markers is limited by a size separation step. The potential of automating SNP marker analysis reduces labour and cost to a minimum. Moreover, the addition of further marker loci easily compensates for the comparatively reduced PIC-values of SNPs. The mean PIC-value of 0.39 determined here for SNPs (Table 3) is, as expected, lower than 0.47 , the average PIC-value of sugar beet markers analysed by SSCP and heteroduplex analysis (Schneider et al. 2001), but it is still higher than the PIC-values for AFLP, RAPD and RFLP markers in soybean (Powell et al. 1996), again indicating the power of SNP mapping. We would also like to stress that the molecular information provided in this paper can easily be adapted and exploited in any other technological platform for SNP detection. 
Applications of multiplexed SNP marker sets in the analysis of segregating populations

Rapid genotyping is a necessity for screening a large amount of DNA samples in a limited time. This is, for example, the case when a phenotypic trait is mapped at high resolution in a large population of individuals. A multiplex SNP marker set of selected linked loci will support rapid and reliable identification of recombination events.

To render the molecular analysis of unrelated traits in a species more efficient a two-step procedure was presented by Roig et al. (2004) for barley. First, markers linked to the phenotypic traits are identified by rapid AFLP genotyping, and then the predictive markers are anchored to a dense map of the genome allowing the precise localization of the genetic locus. In this context, multiplexed LG-specific SNP markers are a valuable tool for anchoring unknown markers to linkage groups.

A further process, which requires rapid repetitive genotyping, is the construction and evaluation of near isogenic lines (Tanksley 1989) to determine the function of a defined fragment of foreign DNA in an otherwise identical background. For this purpose, specific sets of multiplexed SNP markers could accelerate the routine characterization of lines.

\section{Use of multiplexed SNP markers in marker-assisted selection based on specific haplotype-trait associations}

Recently, the existence of robust intragenic haplotypes was revealed in sugar beet (Schneider et al. 2001). This corresponds to the finding that the alleles of a number of linked SNP loci are inherited together defining, on average, from two to four haplotypes per sugar beet gene. In general, one to three SNPs should be sufficient to discriminate between all haplotypes of a locus. The association between a specific haplotype and a phenotypic class has already been demonstrated for the $d$ warf 8 locus of maize (Thornsberry et al. 2001), for six maize genes of the starch metabolism influencing kernel quality (Whitt et al. 2002), and for five DNA markers diagnostic for late blight and plant maturity in potato (Gebhardt et al. 2004). Using predictive SNP alleles of candidate genes in the multiplex format for rapid genotyping, superior lines can be efficiently selected for further breeding.

The protocol developed for multiplexing LG-specific SNP markers is available for adaptation to any of the goals of plant genetics and breeding. For the majority of crop plants, the generation of a tool like the one presented here opens a new era of genotyping which will speed up the development of superior crops.

\section{Acknowledgements}

The authors would like to thank Charlotte Bulich for excellent technical assistance and the members of the MPIZ DNA core facility ADIS for the sequencing work. Plant material and DNA samples provided by KWS SAAT AG and STRUBE-DIECKMANN are greatfully acknowledged. The project was supported by the GABI (Genomanalyse im biologischen System Pflanze) initiative funded by Bundesministerium für Bildung und Forschung (Germany). S.M. acknowledges funding by grant No. 0312283B and K.S. was supported by grant No. 0312283E.

\section{References}

Anderson J.A., Churchill G.A., Autrique J.E., Tanksley S.D. and Sorrells M.E. 1993. Optimizing parental selection for genetic linkage maps. Genome 36: 181-186.

Barzen E., Mechelke W., Ritter E., Seitzer J.F. and Salamini F. 1992. RFLP markers for sugar beet breeding: chromosomal linkage maps and location of major genes for rhizomania resistance, monogermy and hypocotyl colour. Plant J. 2: 601-611.

Barzen E., Mechelke W., Ritter E., Schulte-Kappert E. and Salamini F. 1995. An extended map of the sugar beet genome containing RFLP and RAPD loci. Theor. Appl. Genet. 90: 189193.

Butterfass T. 1964. Die Chloroplastenzahlen in verschiedenartigen Zellen trisomer Zuckerrüben (Beta vulgaris L.). Z. Botanik 52: 46-77.

Cai D., Kleine M., Kifle S., Harloff H.J., Sandal N.N., Marcker K.A., Klein-Lankhorst R.M., Salentijn E.M., Lange W., Stiekema W.J., Wyss U., Grundler F.M. and Jung C. 1997. Positional cloning of a gene for nematode resistance in sugar beet. Science 275: 832-834.

Gebhardt C., Ballvora A., Walkemeier B., Oberhagemann P. and Schüler K. 2004. Assessing genetic potential in germplasm collections of crop plants by marker-trait association: a case study for potatoes with quantitative variation of resistance to late blight and maturity type. Mol. Breeding 13: 93-102.

Hohmann U., Jacobs G., Telgmann A., Gaafar R.M., Alam S. and Jung C. 2003. A bacterial artificial chromosome (BAC) library of sugar beet and a physical map of the region encompassing the bolting gene B. Mol Genet Genomics 269: 126-136.

Hunger S., Di Gaspero G., Möhring S., Bellin D., Schäfer-Pregl R., Borchardt D.C., Durel C.- E., Werber M., Weisshaar B., Salamini F. and Schneider K. 2003. Isolation and linkage analy- 
sis of expressed disease.resistance gene analogues of sugar beet (Beta vulgaris L.). Genome 46: 70-82.

Koornneef M., Hanhart C.J., von Loenen Martinet E.P., Peeters A.J.M., van der Veen J.H. 1987. Arabidopsis Inf. Serv. Frankfurt 23: 46-50.

Liu S., Saha S., Stelly D., Burr B. and Cantrell R.G. 2000. Chromosomal assignment of microsatellite loci in cotton. J. Hered. 91: 326-332.

Makridakis N.M. and Reichardt J.K. 2001. Multiplex automated primer extension analysis: simultaneous genotyping of several polymorphisms. Biotechniques 31: 1374-1380.

Mitchell S.E., Kresovich S., Jester C.A., Hernandez C.J. and Szewc-McFadden A.K. 1997. Application of multiplex PCR and fluorescence-based, semi-automated allele sizing technology for genotyping plant genetic resources. Crop Sci. 37: 617-624.

Narvel J.M., Chu W.- C., Fehr W.R., Cregan P.B. and Shoemaker R.C. 2000. Development of multiplex sets of simple sequence repeat DNA markers covering the soybean genome. Mol. Breed. 6: 175-183.

Nei M. 1987. Molecular evolutionary genetics. Columbia Univ. Press, New York, USA, pp. 512.

Nei M. and Li W.- H. 1979. Mathematical model for studying genetic variation in terms of restriction endonucleases. Proc. Natl. Acad. Sci. USA 76: 5269-5273.

Powell W., Morgante M., Andre C., Hanafey M., Vogel J., Tingey S. and Rafalski A. 1996. The comparison of RFLP, RAPD, AFLP and SSR (microsatellite) markers for germplasm analysis. Mol. Breeding 2: 225-238.

Rafalski A. 2002. Applications of single nucleotide polymorphisms in crop genetics. Curr. Op. Plant Biol. 5: 94-100.

Roig C., Pozzi C., Santi L., Müller J., Stanca M. and Salamini F. 2004. The genetics of barley Hooded suppression. Genetics 167: 439-448.

Schneider K., Borchardt D.C., Schäfer-Pregl R., Nagl N., Glass C., Jeppson A., Gebhardt C. and Salamini F. 1999. PCR-based cloning and segregation analysis of functional gene homologues in Beta vulgaris. Mol. Gen. Genet. 262: 515-524.

Schneider K., Schäfer-Pregl R., Borchardt D.C. and Salamini F. 2002. Mapping QTLs for sucrose content, yield and quality in a sugar beet population fingerprinted by EST-related markers. Theor. Appl. Genet. 104: 1107-1113.

Schneider K., Weisshaar B., Borchardt D.C. and Salamini F. 2001. SNP frequency and allelic haplotype structure of Beta vulgaris expressed genes. Mol. Breeding 8: 63-74.
Schondelmaier J. and Jung C. 1997. Chromosomal assignment of the nine linkage groups of sugar beet (Beta vulgaris L.) using primary trisomics. Theor. Appl. Genet. 95: 590-596.

Schumacher K., Schondelmaier J., Barzen E., Steinruecken G., Borchardt D., Weber W.E., Jung C. and Salamini F. 1997. Combining different linkage maps in sugar beet (Beta vulgaris L.) to make one map. Plant Breeding 116: 23-38.

Stam P. 1993. Construction of integrated genetic linkage maps by means of a new computer package: JoinMap. Plant J. 3: 739 744.

Stam P. and Van Ooijen J. W. 1995. JoinMap (tm) version 2. 0: Software for the calculation of genetic linkage maps. CPRODLO, Wageningen, The Netherlands.

Syvanen A.- C. 2002. Accessing genetic variation: Genotyping single nucleotide polymorphisms. Nature Rev. Genet. 2: 930-942.

Tang S., Kishore V.K. and Knapp S. 2003. PCR-multiplexes for a genome-wide framework of simple sequence repeat marker loci in cultivated sunflower. Theor. Appl. Genet. 107: 6-19.

Tanksley S.D., Grandillo S., Fulton T.M., Zamir D., Eshed Y., Petiard V., Lopez J. and Beckbunn T. 1996. Advanced backcross QTL analysis in a cross between an elite processing line of tomato and its wild relative L. pimpinellifolium. Theor. Appl. Genet. 92: 213-224.

Thornsberry J.M., Goodman M.M., Doebley J., Kresovich S., Nielsen D. and Buckler E.S. 2001. Dwarf8 polymorphisms associate with variation in flowering time. Nat. Genet. 28: 286289.

Törjék O., Berger D., Meyer R.C., Müssig C., Schmid K.J., Rosleff Sörensen T., Weisshaar B., Mitchell-Olds T. and Altmann T. 2003. Establishment of a high-efficiency SNP-based framework marker set for Arabidopsis. Plant J. 36: 122-140.

Van de Peer Y. and De Wachter R. 1994. TREECON for Windows: a software package for the construction and drawing of evolutionary trees for the Microsoft Windows environment. Comput. Applic. Biosci. 10: 569-570.

Voorrips R.E. 2002. MapChart: Software for the graphical presentation of linkage maps and QTLs. The Journal of Heredity 93: 77-78.

Whitt S.R., Wilson L.M., Tenaillon M.I., Gaut B.S. and Buckler E.S. 2002. Genetic diversity and selection in the maize starch pathway. Proc. Nat. Acad. Sci. U.S.A. 99: 12959-12962. 